\title{
Explicit Fermi coordinates and tidal dynamics in de Sitter and Gödel spacetimes
}

\author{
C. Chicone \\ Department of Mathematics, University of Missouri-Columbia, Columbia, Missouri 65211, USA \\ B. Mashhoon \\ Department of Physics and Astronomy, University of Missouri-Columbia, Columbia, Missouri 65211, USA
}

(Received 28 November 2005; published 18 September 2006)

Fermi coordinates are directly constructed in de Sitter and Gödel spacetimes and the corresponding exact coordinate transformations are given explicitly. The quasi-inertial Fermi coordinates are then employed to discuss the dynamics of a free test particle in these spacetimes and the results are compared to the corresponding generalized Jacobi equations that contain only the lowest-order tidal terms. The domain of validity of the generalized Jacobi equation is thus examined in these cases. Furthermore, the difficulty of constructing explicit Fermi coordinates in black hole spacetimes is demonstrated.

DOI: $10.1103 /$ PhysRevD.74.064019

PACS numbers: 04.20.Cv

\section{INTRODUCTION}

To interpret measurements in a gravitational field, access to locally inertial coordinates is indispensable. At each event in spacetime, the spacetime is locally flat; therefore, it is possible to introduce Riemann normal coordinates that constitute a geodesic system of coordinates that is inertial at the event under consideration. The Riemann normal coordinates are in general admissible only in a certain region around the event such that every point in this region can be connected to the event by a unique geodesic.

In general, the physical interpretation of measurements by a free observer necessitates a continuous locally inertial system along the worldline of the observer. This can be achieved by Fermi coordinates, namely, a normal geodesic coordinate system in a cylindrical region about the worldline of the observer. Fermi normal coordinates [1] are the natural extension of Riemann normal coordinates and play a basic role in general relativity theory [2]. The notion of Fermi coordinates was first implicitly introduced in Ref. [1]; a recent commentary on Fermi's pioneering work is contained in Ref. [3].

Imagine a spacetime region with coordinates $x^{\mu}=$ $\left(t, x^{i}\right)$ and a reference observer $O$ following a worldline $\left(\bar{t}, \bar{x}^{i}\right)$. We use gravitational units such that $c=G=1$ throughout; moreover, we assume that the spacetime metric has signature +2 . The observer carries an orthonormal parallel-propagated tetrad frame $\lambda^{\mu}{ }_{(\alpha)}$ along its path such that $\lambda^{\mu}{ }_{(0)}=d \bar{x}^{\mu} / d \tau$, where $\tau$ is the proper time along the worldline of $O$. Thus $\lambda^{\mu}{ }_{(0)}$ is the timelike unit vector that is tangent to the worldline of $O$ and acts as its local temporal axis. Moreover, $\lambda_{(i)}^{\mu}, i=1,2,3$, are orthogonal spacelike unit gyro axes that form the local spatial frame of the observer. At each event $Q(\tau)$ along the worldline, consider the class of spacelike geodesics orthogonal to the worldline; these form a local hypersurface. Let $P$ be an event with coordinates $x^{\mu}$ on this hypersurface and consider the unique spacelike geodesic segment from $Q$ to $P$. We define the Fermi coordinates of $P$ to be $X^{\mu}=\left(T, X^{i}\right)$, where

$$
T=\tau, \quad X^{i}=\sigma \xi^{\mu} \lambda_{\mu}{ }^{(i)} .
$$

Here $\xi^{\mu}$ is the unit vector tangent to the spacelike geodesic segment at $Q$ and $\sigma$ is the proper length of this segment from $Q$ to $P$ as in Fig. 1. Thus the reference observer $O$ is always at the spatial origin of Fermi coordinates.

The Fermi coordinates are in general admissible in the sense of Lichnerowicz [4] in a finite cylindrical region about the worldline of $O$. The spacetime metric in Fermi coordinates is given by

$$
\begin{gathered}
g_{00}=-1-{ }^{F} R_{0 i 0 j}(T) X^{i} X^{j}+\cdots, \\
g_{0 i}=-\frac{2}{3}{ }^{F} R_{0 j i k}(T) X^{j} X^{k}+\cdots,
\end{gathered}
$$

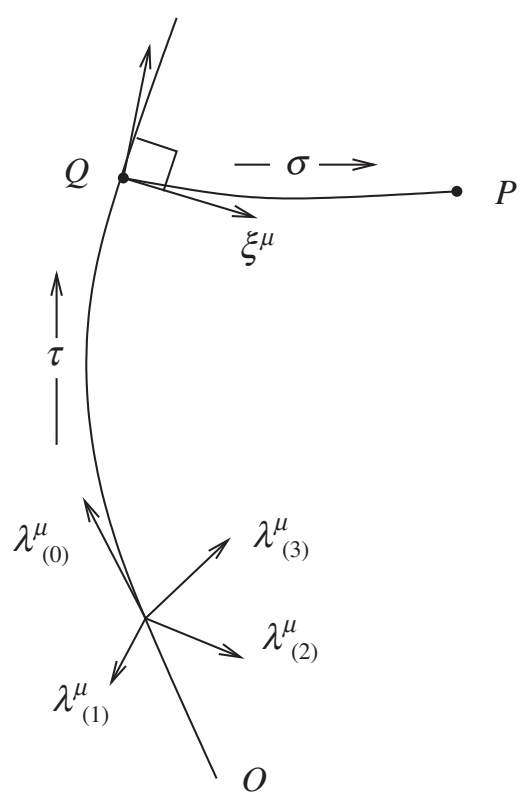

FIG. 1. Schematic construction of Fermi coordinates at $P$. 


$$
g_{i j}=\delta_{i j}-\frac{1}{3} F R_{i k j l}(T) X^{k} X^{l}+\cdots,
$$

where

$$
{ }^{F} R_{\alpha \beta \gamma \delta}(T)=R_{\mu \nu \rho \sigma} \lambda^{\mu}{ }_{(\alpha)} \lambda^{\nu}{ }_{(\beta)} \lambda^{\rho}{ }_{(\gamma)} \lambda^{\sigma}{ }_{(\delta)}
$$

is the projection of the Riemann tensor on the observer's tetrad along the reference trajectory. The Taylor series in Eqs. (2)-(4) can be expressed as $g_{\mu \nu}=\eta_{\mu \nu}+h_{\mu \nu}(X)$, where $h_{\mu \nu}(X)$ is a perturbation that can be expressed as a series expansion in powers of the spatial distance away from the reference trajectory. The nature of the infinite series in Eqs. (2)-(4) has been discussed by a number of authors. The second-order terms given explicitly in Eqs. (2)-(4) were worked out in Refs. [5,6]. The thirdorder terms were first given in Ref. [7] and the fourth-order terms in Ref. [8]; moreover, these higher-order terms have been recently discussed in Ref. [9]. In the weak-field limit, the infinite series in Eqs. (2)-(4) have been studied in Ref. [10].

Consider the motion of a free particle in the Fermi coordinate system. The geodesic equation of motion

$$
\frac{d U^{\mu}}{d s}+\Gamma_{\rho \sigma}^{\mu} U^{\rho} U^{\sigma}=0,
$$

where $U^{\mu}=d X^{\mu} / d s$, can be written in terms of the modified Lorentz factor $\Gamma=d T / d s$ and $\mathbf{V}=d \mathbf{X} / d T$ based on the decomposition $U^{\mu}=\Gamma(1, \mathbf{V})$. On the other hand, the timelike condition $U^{\mu} U_{\mu}=-1$ implies that

$$
-\frac{1}{\Gamma^{2}}=g_{00}+2 g_{0 i} V^{i}+g_{i j} V^{i} V^{j} .
$$

The equation for $\mathbf{V}(T)$ is the reduced geodesic equation given by

$$
\frac{d V^{i}}{d T}+\left(\Gamma_{\alpha \beta}^{i}-\Gamma_{\alpha \beta}^{0} V^{i}\right) \frac{d X^{\alpha}}{d T} \frac{d X^{\beta}}{d T}=0 .
$$

It is useful to consider the class of static observers in the Fermi coordinate system. These observers are generally accelerated with four-velocity $U_{S}^{\mu}=\left(-g_{00}\right)^{-(1 / 2)} \delta^{\mu}{ }_{0}$ in Fermi coordinates. The Lorentz factor of the test particle with four-velocity $U^{\mu}=\Gamma(1, \mathbf{V})$ with respect to the static observers is

$$
\Gamma_{S}=\left(\sqrt{-g_{00}}-\frac{g_{0 i} V^{i}}{\sqrt{-g_{00}}}\right) \Gamma .
$$

In this paper, we are interested in the comparison between the consequences of these equations in the case of exact Fermi coordinates - given explicitly in de Sitter and Gödel spacetimes - with equations based on the lowestorder tidal terms given explicitly in Eqs. (2)-(4). In the latter case, Eq. (7) implies that

$$
\begin{aligned}
\frac{1}{\Gamma^{2}}= & 1-V^{2}+{ }^{F} R_{0 i 0 j} X^{i} X^{j}+\frac{4}{3}{ }^{F} R_{0 j i k} X^{j} V^{i} X^{k} \\
& +\frac{1}{3}{ }^{F} R_{i k j l} V^{i} X^{k} V^{j} X^{l},
\end{aligned}
$$

and the reduced equation of motion is the generalized Jacobi equation [11]

$$
\begin{gathered}
\frac{d^{2} X^{i}}{d T^{2}}+{ }^{F} R_{0 i 0 j} X^{j}+2^{F} R_{i k j 0} V^{k} X^{j}+\frac{2}{3}\left(3^{F} R_{0 k j 0} V^{i} V^{k}\right. \\
\left.+{ }^{F} R_{i k j l} V^{k} V^{l}+{ }^{F} R_{0 k j l} V^{i} V^{k} V^{l}\right) X^{j}=0 .
\end{gathered}
$$

This equation is expected to be valid for $|\mathbf{X}|$ sufficiently small compared to a certain radius of curvature of spacetime. We assume the following initial conditions for Eqs. (8) and (11) throughout this paper: At $T=0, \mathbf{X}=$ 0 and $\mathbf{V}=\mathbf{V}_{0}$ such that $\left|\mathbf{V}_{0}\right|<1$. Let us note that for $T>$ 0 and away from the origin of Fermi coordinates, $|\mathbf{V}|$ could then in principle exceed unity along a timelike geodesic worldline.

In some situations - for instance, along the axis of rotational symmetry of a Kerr black hole-one-dimensional motion is allowed. In general, the symmetries of the Riemann tensor imply that for motion along the $Z$ direction, say, Eq. (11) reduces to

$$
\frac{d^{2} Z}{d T^{2}}+\kappa\left(1-2 \dot{Z}^{2}\right) Z=0,
$$

where $\kappa(T)={ }^{F} R_{T Z T Z}(T)$ and $\dot{Z}=d Z / d T$. This equation approximates the reduced geodesic equation for $|Z|$ sufficiently small compared to $|\kappa|^{-1 / 2}$. In Eq. (12), the critical speed of $1 / \sqrt{2} \approx 0.7$ should be noted: Motion at the critical speed is uniform, i.e. $Z=$ constant $\pm T / \sqrt{2}$, and the character of the motion changes depending on whether the initial relative speed is above or below the critical speed. The critical speed in gravitational motion is briefly reviewed in Appendix A.

In a recent series of papers, we have investigated the general equation of motion of a particle in Fermi coordinates using the generalized Jacobi equation [11]. The results are particularly interesting if the speed of the test particle relative to the reference particle exceeds the critical speed given by $V_{c}:=1 / \sqrt{2}$. The astrophysical implications of these results have been worked out in Refs. [12,13]. This general approach has been extended to the motion of charged particles in Ref. [14]. A major shortcoming of these studies is that only the first few terms of the series in Eqs. (2)-(4) have been taken into account. It is therefore important to know the domain of validity of these astrophysically significant results. In fact, as explicitly demonstrated in Appendix B, the construction of the exact Fermi coordinates in black hole spacetimes is a daunting task. Moreover, the transformation $x^{\mu} \mapsto X^{\mu}$ remains implicit as well as approximate in these studies [1113]. For recent efforts to construct such approximate transformations explicitly, see Ref. [15]. 
To avoid the difficulties encountered in black hole spacetimes (cf. Appendix B), we turn to spacetimes with more symmetries. It is clear that explicit Fermi coordinates can only be constructed in rather special circumstances; however, the generalized Jacobi equation can be employed in general. What is the extent of agreement between these approaches? To answer this question, we choose two spatially homogeneous spacetimes: de Sitter's spacetime with ten Killing vector fields and Gödel's spacetime with five Killing vector fields.

The main purpose of the present paper is to study the infinite series in Eqs. (2)-(4) and the exact transformation $x^{\mu} \mapsto X^{\mu}$ to Fermi coordinates explicitly in de Sitter and Gödel spacetimes. The motion of a test particle is then studied using the exact Fermi metric and the results are compared to the implications of the generalized Jacobi Eq. (12), where in de Sitter (Gödel) spacetime $\kappa$ is a negative (positive) constant. Equation (12) can be integrated in these cases; the behavior of the solutions have been studied in Ref. [11]. The comparison of the exact geodesic equation of motion in Fermi coordinates with the generalized Jacobi equation makes it in principle possible to determine the extent of validity of the latter equation in these cases; moreover, a general mathematical treatment of such a comparison is presented in Appendix C.

\section{FERMI COORDINATES IN DE SITTER SPACETIME}

Consider de Sitter's metric in the (inflationary-model) form

$$
d s^{2}=-d t^{2}+\mathcal{A}^{2}(t) \delta_{i j} d x^{i} d x^{j},
$$

where $\mathcal{A}(t)$ is given by

$$
\mathcal{A}(t)=e^{H t},
$$

and $H>0$ is a ("Hubble") constant. This metric satisfies Einstein's matter-free equations such that $H^{2}=\Lambda / 3$, where $\Lambda>0$ is the cosmological constant. The spacetime region $x^{\mu}=(t, \mathbf{x})$ described by Eq. (1) is only part of de Sitter's spacetime of constant positive curvature; a detailed historical description of spacetimes of constant curvature is contained in Ref. [16].

The geodesics of the metric (13) are obtained from the extrema of $\int d s$. It is a simple consequence of this fact that $\mathcal{A}^{2} d x^{i} / d s, i=1,2,3$, are constants along the geodesics. Let us imagine a class of reference observers that are fixed in space, i.e. they have constant spatial coordinates $x^{i}, i=$ $1,2,3$. It turns out that these are free observers each with an orthonormal tetrad frame $\lambda_{(\alpha)}^{\mu}$ such that

$$
\lambda^{\mu}{ }_{(0)}=\delta^{\mu}{ }_{0}, \quad \lambda^{\mu}{ }_{(i)}=\frac{1}{\mathcal{A}(t)} \delta^{\mu},
$$

where $t=\tau$ is the proper time along the worldline of the observer. Moreover, it is simple to verify that this tetrad frame is parallel transported along the geodesic worldline of the observer.

The projection of the Riemann tensor on the tetrad of a reference observer can be expressed as a $6 \times 6$ matrix $\mathcal{R}=\left(\mathcal{R}_{A B}\right)$, where $A$ and $B$ are elements of the set $\{01,02,03,23,31,12\}$. We find that

$$
\mathcal{R}=-H^{2}\left[\begin{array}{cc}
I & 0 \\
0 & -I
\end{array}\right],
$$

where $I$ is the $3 \times 3$ unit matrix.

We need to find the general solution for spacelike geodesics. To this end, the spacelike geodesic equation in this case reduces to

$$
\begin{gathered}
\frac{d x^{i}}{d \sigma}=\frac{C_{i}}{\mathcal{A}^{2}(t)}, \\
\left(\frac{d t}{d \sigma}\right)^{2}=\frac{C^{2}}{\mathcal{A}^{2}(t)}-1,
\end{gathered}
$$

where $\sigma$ is the proper length along a spacelike geodesic, $C_{i}, i=1,2,3$, are constants, and

$$
C^{2}=\sum_{i} C_{i}^{2} .
$$

The general solution of Eq. (18) is

$$
e^{H t}=C \cos (H \sigma+\eta),
$$

where $\eta$ is a constant. It follows from Eq. (20) that Eq. (17) can be solved exactly and the result is

$$
x^{i}=\frac{C_{i}}{H C^{2}} \tan (H \sigma+\eta)+D_{i},
$$

where $D_{i}, i=1,2,3$, are constants of integration.

To establish a Fermi coordinate system, we need to choose a specific reference trajectory. For the sake of simplicity and with no loss in generality, we choose the reference observer $O:(\bar{t}, \overline{\mathbf{x}})=(\tau, \mathbf{0})$. In the construction of Fermi coordinates (see Fig. 1), the spacelike geodesic segment from $Q$ to $P$ is given by Eqs. (20) and (21) such that $\sigma=0$ at $Q$. Therefore,

$$
e^{H \tau}=C \cos \eta, \quad \frac{C_{i}}{H C^{2}} \tan \eta+D_{i}=0 .
$$

Moreover, $\xi^{\mu}$ is the vector tangent to the spacelike geodesic segment at $Q$ and is given in $(t, \mathbf{x})$ coordinates by

$$
\xi^{\mu}=\left(-\tan \eta, \frac{C_{i}}{C^{2} \cos ^{2} \eta}\right) .
$$

It follows from $\xi_{\mu} \lambda^{\mu}{ }_{(0)}=0$ that $\tan \eta=0$ and hence $D_{i}=0, i=1,2,3$, from Eq. (22). We choose $\eta=0$ for simplicity; hence, $C=\exp (H \tau)$. Finally, we compute the spatial Fermi coordinates of $P$ using Eq. (1). It follows that event $P$ has Fermi coordinates

$$
T=\tau, \quad \mathbf{X}=\sigma e^{-H \tau} \mathbf{C} .
$$


It is useful to recognize that $\sigma$ is the radial Fermi coordinate $R$,

$$
R=\sqrt{\delta_{i j} X^{i} X^{j}},
$$

so that we find from Eqs. (20) and (21) that the transformation $(t, \mathbf{x}) \mapsto(T, \mathbf{X})$ is given by

$$
\begin{gathered}
e^{H t}=e^{H T} \cos (H R), \\
\mathbf{x}=e^{-H T} \frac{\tan (H R)}{H R} \mathbf{X} .
\end{gathered}
$$

De Sitter's metric in Fermi coordinates is $d s^{2}=$ $g_{\mu \nu} d X^{\mu} d X^{\nu}$, where

$$
\begin{gathered}
g_{00}=-\cos ^{2}(H R), \quad g_{0 i}=0, \\
g_{i j}=\frac{X^{i} X^{j}}{R^{2}}+\frac{\sin ^{2}(H R)}{H^{2} R^{2}}\left(\delta_{i j}-\frac{X^{i} X^{j}}{R^{2}}\right) .
\end{gathered}
$$

The Fermi coordinates cover a static spacetime region and are admissible for $0 \leq R<\pi /(2 H)$; in fact, $g_{00}=0$ for $H R=\pi / 2$.

The form of the metric (28) and (29) can be simplified if we introduce Fermi polar coordinates via

$$
\begin{gathered}
X^{1}=R \sin \theta \cos \phi, \quad X^{2}=R \sin \theta \sin \phi, \\
X^{3}=R \cos \theta .
\end{gathered}
$$

The Fermi metric in these spherical polar coordinates is given by

$$
\begin{aligned}
d s^{2}= & -\cos ^{2}(H R) d T^{2}+d R^{2} \\
& +\frac{1}{H^{2}} \sin ^{2}(H R)\left(d \theta^{2}+\sin ^{2} \theta d \phi^{2}\right) .
\end{aligned}
$$

It is interesting to note that this form of de Sitter's metric already appeared in de Sitter's original investigations, namely, in his 1917 paper on the curvature of space [cf. Eq. (2.5) of Ref. [16] and the discussion therein].

Writing $\cos ^{2}(H R)$ and $\sin ^{2}(H R)$ in Eqs. (28) and (29) in terms of $\cos (2 H R)$ and the subsequent Taylor expansion of $\cos (2 H R)$ about $R=0$ would lead to the standard series expansion of the elements of the metric tensor in Fermi coordinates as in Eqs. (2)-(4). Such series are uniformly convergent for all $R$; however, the Fermi coordinates are admissible only for $0 \leq 2 H R<\pi$. The hypersurface $R=$ $\pi /(2 H)$, where the timelike Killing vector $\partial_{T}$ becomes null, is a static limit surface.

\section{TIDAL DYNAMICS IN DE SITTER SPACETIME}

Let us now consider the radial motion of a test particle away from the reference observer $O$ at $R=0$ in the spherical Fermi coordinates (30). We concentrate on the general reduced geodesic Eq. (8). In the case of radial motion in the spherically symmetric spacetime region of Eq. (31), the only relevant nonzero connection coefficients are

$$
\Gamma_{T T}^{R}=-\frac{1}{2} H \sin (2 H R), \quad \Gamma_{T R}^{T}=\Gamma_{R T}^{T}=-H \tan (H R) .
$$

Therefore, the radial equation of motion is

$$
\frac{d^{2} R}{d T^{2}}-H \tan (H R)\left[\cos ^{2}(H R)-2\left(\frac{d R}{d T}\right)^{2}\right]=0 .
$$

This equation can be integrated easily once and the result for $\dot{R}=d R / d T$ is

$$
\dot{R}^{2}=\cos ^{2}(H R)-\left(1-V_{0}^{2}\right) \cos ^{4}(H R),
$$

where $V_{0}=\dot{R}(T=0)$ is the initial speed at $R=0$. Next, reducing Eq. (34) to quadrature and using the relation

$$
\begin{aligned}
\int \frac{d x}{\cos x \sqrt{1+\lambda^{2} \sin ^{2} x}=} & \frac{1}{2 \sqrt{1+\lambda^{2}}} \\
& \times \ln \frac{\sqrt{1+\lambda^{2}} \sin ^{2} x}{\sqrt{1+\lambda^{2}} \sin ^{2} x}-\sqrt{1+\lambda^{2}} \sin x \\
& =\sqrt{1+\lambda^{2}} \sin x
\end{aligned}
$$

with $\lambda^{2}=-1+1 / V_{0}^{2}$, it is possible to show that the general solution of the radial equation with the specified initial conditions is

$$
\tan (H R)= \pm V_{0} \sinh (H T) .
$$

In general, we require that the Fermi time $T$ increase along the timelike worldline of every observer. Therefore, assuming $V_{0}>0$, we are only interested in the upper sign in Eq. (36). The modified Lorentz factors $\Gamma$ and $\Gamma_{S}$ are given in this case by

$$
\Gamma=\frac{\Gamma_{0}}{\cos ^{2}(H R)}, \quad \Gamma_{S}=\frac{\Gamma_{0}}{\cos (H R)},
$$

where $\Gamma_{0}=\left(1-V_{0}^{2}\right)^{-(1 / 2)}$. The speed of the particle initially increases, remains constant or decreases depending upon whether $V_{0}$ is less than, equal to, or greater than the critical speed $1 / \sqrt{2}$; nevertheless, Eq. (37) indicates that regardless the particle's initial speed, as $T \rightarrow \infty$ it monotonically approaches a null geodesic at the static limit surface $H R=\pi / 2$. Moreover, the speed of the particle, as "seen" by the reference observer, is zero at this boundary surface, where everything, including light, "appears frozen." We note that $\dot{R}$ has a maximum at $\cos (H R)=$ $\Gamma_{0} / \sqrt{2}$ for $V_{0} \leq 1 / \sqrt{2}$ as illustrated in Fig. 2. The case of a null ray follows from Eq. (36) with the upper sign for $V_{0}=$ 1; in fact, in this case Eq. (36) also can be expressed as $\tanh (H T)=\sin (H R)$.

Let us now determine to what extent these exact results are reflected in the first-order tidal terms of the generalized Jacobi Eq. (11). For radial motion, we find

$$
\frac{d^{2} R}{d T^{2}}=H^{2}\left(1-2 \dot{R}^{2}\right) R,
$$



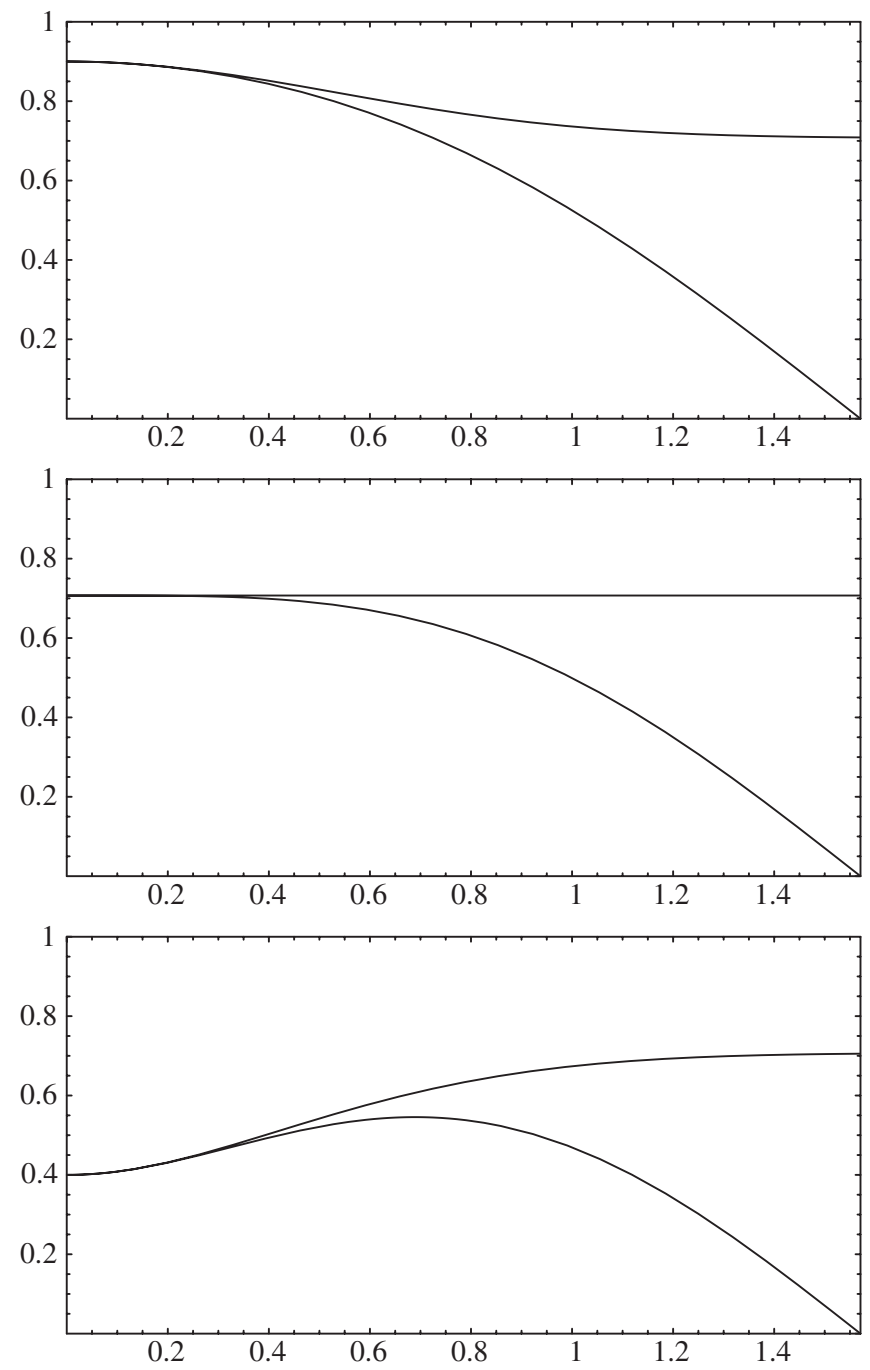

FIG. 2. Plot of $\dot{R}$ versus $H R, 0 \leq H R \leq \pi / 2$, for $V_{0}=0.4$, $1 / \sqrt{2}$ and 0.9 . In each panel, the lower (upper) curve represents the motion according to the exact (approximate) equation of motion in Fermi coordinates. In the exact case, $\dot{R}$ vanishes at $H R=\pi / 2$.

which follows as well from Eq. (33), since to linear order we have $\tan (H R) \approx H R$ and $\cos (H R) \approx 1$. Equation (38) is thus valid for $R$ sufficiently small compared to $H^{-1}$.

To study the general behavior of the solution of Eq. (38), we note that this equation can be integrated once and the result is

$$
\dot{R}^{2}=V_{c}^{2}-\left(V_{c}^{2}-V_{0}^{2}\right) e^{-2 H^{2} R^{2}},
$$

where $V_{c}=1 / \sqrt{2}$. Let us define a function $\Psi_{ \pm}(x ; \nu)$ via the integral

$$
\Psi_{ \pm}(x ; \nu):=\int_{0}^{x} \frac{d z}{\sqrt{1+\nu e^{ \pm z^{2}}}}
$$

where $\nu$ is a constant parameter. Near $x=0$, we have the Taylor expansions

$$
\begin{gathered}
\Psi_{ \pm}(x ; \nu)=(\nu+1)^{-1 / 2}\left[x \mp \frac{\nu}{\nu+1} \frac{x^{3}}{3 !}\right. \\
\left.+\frac{3 \nu(\nu-2)}{(\nu+1)^{2}} \frac{x^{5}}{5 !}+\cdots\right], \\
x=(\nu+1)^{1 / 2}\left[\Psi_{ \pm} \pm \frac{\nu}{3 !} \Psi_{ \pm}^{3}+\frac{\nu(7 \nu+6)}{5 !} \Psi_{ \pm}^{5}+\cdots\right] .
\end{gathered}
$$

The general solution of Eq. (39) is thus given by

$$
\Psi_{-}\left(\sqrt{2} H R ; 2 V_{0}^{2}-1\right)= \pm H T .
$$

For the physical problem under consideration here only the upper sign in Eq. (43) is needed; in this case, the motion can be described in terms of an attractor involving uniform motion at speed $V_{c}$. Explicitly, it is simple to see via the effective potential in Eq. (39) that for $V_{0}>V_{c}$, the particle monotonically decelerates and asymptotically (i.e. for $H T \rightarrow \infty$ and $H R \rightarrow \infty)$ approaches the critical speed. For $V_{0}=V_{c}$, the particle moves uniformly, while for $V_{0}<$ $V_{c}$ the particle monotonically accelerates and asymptotically approaches $V_{c}$. These results, together with the extent of the (initial) agreement between Eq. (43) and the exact result given by Eq. (36), are illustrated in Fig. 2. Analytic estimates for the difference between the exact solution and the approximation at a given time $T$ can be obtained using Eqs. (36) and (43).

\section{FERMI COORDINATES IN GÖDEL SPACETIME}

Let us next consider the stationary and spatially homogeneous rotating universe model discovered by Gödel [17]. It has been discussed by a number of authors [18]. Gödel's metric can be expressed as

$$
d s^{2}=-d t^{2}-2 \sqrt{2} U(x) d t d y+d x^{2}-U^{2} d y^{2}+d z^{2},
$$

where

$$
U(x)=e^{\sqrt{2} \Omega x}
$$

and $\Omega$ is a positive constant. The Ricci curvature for this spacetime is given by

$$
R_{\mu \nu}=2 \Omega^{2} u_{\mu} u_{\nu},
$$

where $u^{\mu}=\delta^{\mu}{ }_{0}$ is the four-velocity vector field for free particles that are at rest in space and coincides with the timelike Killing vector field $\partial_{t}$. The source of the Gödel gravitational field could be a perfect fluid with velocity $u^{\mu}$ and constant density $\mu$ and pressure $\tilde{p}$ given by $\mu=\tilde{p}=$ $\Omega^{2} /(8 \pi)$, where $\Omega \partial_{z}$ is the vorticity vector of the geodesic worldlines of the fluid source. Alternatively, the source of the Gödel field could be dust of constant density $\Omega^{2} /(4 \pi)$ together with a cosmological constant $\Lambda=-\Omega^{2}$ [19]. The investigation of the physical aspects of the Gödel universe 
has led to the introduction of various interesting coordinate systems [20,21]; for instance, Gaussian coordinate systems for the Gödel spacetime have been constructed in Ref. [20].

As in Sec. II, we are interested in the class of reference observers that are fixed in space; that is, they have constant $x, y$, and $z$ coordinates. These free observers are endowed with an orthonormal tetrad frame $\lambda^{\mu}{ }_{(\alpha)}$ that is parallel transported along their geodesic worldlines. Thus $\lambda^{\mu}{ }_{(0)}=$ $u^{\mu}$ and $\lambda_{(i)}^{\mu}, i=1,2,3$, can be expressed as

$$
\begin{gathered}
\lambda_{(1)}^{\mu}=\tilde{\lambda}_{(1)}^{\mu} \cos \Omega t+\tilde{\lambda}_{(2)}^{\mu} \sin \Omega t, \\
\lambda_{(2)}^{\mu}=-\tilde{\lambda}_{(1)}^{\mu} \sin \Omega t+\tilde{\lambda}_{(2)}^{\mu} \cos \Omega t,
\end{gathered}
$$

where in $(t, x, y, z)$ coordinates we have $\tilde{\lambda}_{(1)}^{\mu}=\delta^{\mu}{ }_{1}$ and

$$
\tilde{\lambda}_{(2)}^{\mu}=\left(-\sqrt{2}, 0, \frac{1}{U(x)}, 0\right) ;
$$

moreover, $\lambda_{(3)}^{\mu}=\delta^{\mu}{ }_{3}$ coincides with the spacelike Killing vector field $\partial_{z}$. Thus at each point in space the unit gyro axes of the corresponding reference observer rotate about the $z$ direction with frequency $\Omega$. For the sake of simplicity and without any loss in generality, we choose the reference observer at $x=y=z=0$.

It can be shown that all of the nonzero components of the Riemann tensor for metric (44) can be obtained from

$$
\begin{aligned}
& R_{0101}=\Omega^{2}, \quad R_{0202}=\Omega^{2} U^{2}, \\
& R_{0112}=-\sqrt{2} \Omega^{2} U, \quad R_{1212}=3 \Omega^{2} U^{2},
\end{aligned}
$$

via the symmetries of the Riemann tensor. It is then straightforward to demonstrate that the nonzero components of the projection of the Riemann tensor on the reference tetrad field can be obtained from

$$
{ }^{F} R_{0101}={ }^{F} R_{0202}={ }^{F} R_{1212}=\Omega^{2}
$$

via the symmetries of the Riemann tensor. Using Eqs. (2)(4), we note that in Fermi coordinates the spacetime metric - containing only the lowest-order tidal terms - would be

$$
\begin{aligned}
d s^{2} \approx & -\left[1+\Omega^{2}\left(X^{2}+Y^{2}\right)\right] d T^{2}+d X^{2}+d Y^{2}+d Z^{2} \\
& -\frac{1}{3} \Omega^{2}(X d Y-Y d X)^{2} .
\end{aligned}
$$

To find the exact expression for this metric, we need to specify all of the spacelike geodesics of the Gödel spacetime that are orthogonal to the worldline of the chosen reference observer $O:(\bar{t}, \overline{\mathbf{x}})=(\tau, \mathbf{0})$. The spacelike geodesics of the Gödel spacetime are given by

$$
\begin{gathered}
t^{\prime}+\sqrt{2} U y^{\prime}=E, \quad \sqrt{2} U t^{\prime}+U^{2} y^{\prime}=k, \\
z^{\prime}=h, \quad-t^{\prime 2}-2 \sqrt{2} U t^{\prime} y^{\prime}+x^{\prime 2}-U^{2} y^{\prime 2}+z^{\prime 2}=1,
\end{gathered}
$$

where $t^{\prime}=d t / d \sigma$, etc. Here $\sigma$ is the proper length of the spacelike geodesic and $E, k$, and $h$ are constants of integration. We need a general solution of these equations that would correspond to the geodesic segment from $Q$ to $P$ in Fig. 1. The vector tangent to this segment, namely, $\left(t^{\prime}, x^{\prime}, y^{\prime}, z^{\prime}\right)$ at $\sigma=0$ is $\xi^{\mu}$ at event $Q:(\tau, \mathbf{0})$; moreover, $\xi^{\mu}$ is orthogonal to $\lambda_{(0)}^{\mu}$, i.e.

$$
-\xi_{\mu} \lambda_{(0)}^{\mu}=t^{\prime}+\sqrt{2} U y^{\prime}=E=0,
$$

where Eq. (53) has been used. Therefore, with $E=0$ and $z=h \sigma$, Eqs. (53) and (54) imply that

$$
t^{\prime}=\sqrt{2} \frac{k}{U}, \quad x^{\prime 2}+\frac{k^{2}}{U^{2}}=1-h^{2}, \quad y^{\prime}=-\frac{k}{U^{2}},
$$

where $h^{2} \leq 1$. The equation for $x$ can be written as

$$
U^{\prime 2}-2\left(1-h^{2}\right) \Omega^{2} U^{2}=-2 \Omega^{2} k^{2},
$$

which has the general solution

$$
U=\alpha \cosh (a \sigma+b),
$$

where $a:=\Omega \sqrt{2\left(1-h^{2}\right)}$. Here $\alpha>0$ and $b$ are constants that are related by the requirement that $U=1$ at $\sigma=0$, i.e.

$$
\alpha \cosh b=1 .
$$

Substitution of Eq. (58) in Eq. (57) results in

$$
\alpha a=\sqrt{2} \Omega|k| .
$$

It follows from the solution of the other equations in (56) that

$$
\begin{array}{r}
t-\tau=\frac{2 k}{\Omega|k|}\left(\arctan e^{a \sigma+b}-\arctan e^{b}\right), \\
y=-\frac{k}{\sqrt{2} \alpha \Omega|k|}[\tanh (a \sigma+b)-\tanh b] .
\end{array}
$$

Next, we compute $\xi_{\mu} \lambda^{\mu}{ }_{(i)}$ at $Q$, where $\sigma=0$, and use Eq. (1) to find Fermi coordinates $(T, X, Y, Z)$ such that

$$
\begin{gathered}
T=\tau, \quad X \cos \Omega T-Y \sin \Omega T=\sigma|k| \sinh b, \\
X \sin \Omega T+Y \cos \Omega T=-\sigma k, \quad Z=\sigma h .
\end{gathered}
$$

We note that $X^{2}+Y^{2}+Z^{2}=\sigma^{2}$, since $h^{2}+k^{2} / \alpha^{2}=1$. It proves useful to introduce cylindrical Fermi coordinates $(\rho, \varphi, Z)$ such that

$$
X=\rho \cos \varphi, \quad Y=\rho \sin \varphi .
$$

Then, Eqs. (63) and (64) can be written as

$$
\begin{gathered}
\sqrt{2} \Omega \rho=a \sigma, \quad \cos (\varphi+\Omega T)=\tanh b, \\
\sin (\varphi+\Omega T)=-\frac{k}{|k|} \alpha .
\end{gathered}
$$


Consider now Eq. (58) for $x$ : expanding its right-hand side and using Eq. (66) together with Eq. (59), we find

$$
e^{\sqrt{2} \Omega x}=\cosh (\sqrt{2} \Omega \rho)+\sinh (\sqrt{2} \Omega \rho) \cos (\varphi+\Omega T) .
$$

Similarly, Eq. (62) for $y$ can be written as

$$
\sqrt{2} \Omega y=\frac{\tanh (\sqrt{2} \Omega \rho) \sin (\varphi+\Omega T)}{1+\tanh (\sqrt{2} \Omega \rho) \cos (\varphi+\Omega T)},
$$

while Eq. (61) for $t-T$ takes the form

$$
\begin{aligned}
\tan & {\left[\frac{1}{2} \Omega(T-t)\right] } \\
& =\frac{\left(e^{\sqrt{2} \Omega \rho}-1\right) \sin (\varphi+\Omega T)}{1-\cos (\varphi+\Omega T)+[1+\cos (\varphi+\Omega T)] e^{\sqrt{2} \Omega \rho}} .
\end{aligned}
$$

It is advantageous to introduce new variables $u$ and $v$ by

$$
u=\sqrt{2} \Omega \rho, \quad v=\varphi+\Omega T,
$$

so that the transformation to Fermi coordinates, $(t, x, y, z) \mapsto(T, X, Y, Z)$, is given by

$$
\begin{gathered}
\tan \left[\frac{1}{2} \Omega(T-t)\right]=\frac{\left(e^{u}-1\right) \sin v}{1-\cos v+(1+\cos v) e^{u}}, \\
e^{\sqrt{2} \Omega x}=\cosh u+\cos v \sinh u, \\
\sqrt{2} \Omega y=\frac{\sinh u \sin v}{\cosh u+\sinh u \cos v}, \quad z=Z .
\end{gathered}
$$

It should be noted that transformations (72) and (73) bear a strong resemblance to those used by Gödel to show the rotational symmetry of his metric about the $z$ axis (see Ref. [17], p. 449).

One can show that

$$
\begin{aligned}
& d t+\sqrt{2} U d y=d T+\frac{1}{\Omega}(\cosh u-1) d v, \\
& d x^{2}+U^{2} d y^{2}=\frac{1}{2 \Omega^{2}}\left(d u^{2}+\sinh ^{2} u d v^{2}\right),
\end{aligned}
$$

so that the Gödel metric

$$
d s^{2}=-(d t+\sqrt{2} U d y)^{2}+d x^{2}+U^{2} d y^{2}+d z^{2},
$$

now takes the form

$$
\begin{aligned}
d s^{2}= & -\left[d T+\frac{1}{\Omega}(\cosh u-1) d v\right]^{2} \\
& +\frac{1}{2 \Omega^{2}}\left(d u^{2}+\sinh ^{2} u d v^{2}\right)+d Z^{2}
\end{aligned}
$$

in $(T, u, v, Z)$ coordinates. From $\rho d \rho=X d X+Y d Y$ and $\rho^{2} d \varphi=X d Y-Y d X$, the form of the metric in Fermi coordinates $(T, X, Y, Z)$ is

$$
\begin{aligned}
d s^{2}= & -(1+L) d T^{2}-2 \Omega F d T(X d Y-Y d X) \\
& +d X^{2}+d Y^{2}+d Z^{2}+\frac{\mathcal{H}}{\rho^{2}}(X d Y-Y d X)^{2},
\end{aligned}
$$

where $\rho=\sqrt{X^{2}+Y^{2}}, u=\sqrt{2} \Omega \rho$,

$$
\begin{gathered}
L(u)=\frac{1}{2} \sinh ^{2} u, \quad F(u)=\left(\frac{\cosh u-1}{u}\right)^{2}, \\
\mathcal{H}(u)=\left(\frac{\sinh u}{u}\right)^{2}-1-2 F(u) .
\end{gathered}
$$

The metric functions $L, F$, and $\mathcal{H}$ depend upon $\cosh u$ and $\cosh (2 u)$; expanding these in uniformly convergent power series in $u=\sqrt{2} \Omega \rho$ for any $\rho \geq 0$, we find the complete tidal expansion of the metric in Fermi coordinates. This result agrees with Eq. (52) to order $\Omega^{2}$.

To examine the admissibility of Fermi coordinates in the sense of Lichnerowicz [4], we need to determine both $\left(g_{\mu \nu}\right)$ and $\left(g^{\mu \nu}\right)$ in Fermi coordinates. Indeed, given our convention that the signature of the metric is +2 , Lichnerowicz admissibility requires that the principal minors of these matrices be negative. We recall that for a symmetric $n \times n$ matrix $M$, the principal minors are defined by

$$
\operatorname{det}\left[\begin{array}{ccc}
M_{11} & \cdots & M_{1 k} \\
\vdots & & \vdots \\
M_{k 1} & \cdots & M_{k k}
\end{array}\right]
$$

for $k=1, \ldots, n$. Employing $(T, v, \rho, Z)$ coordinates, it is possible to show that the admissibility conditions for the inverse metric are satisfied provided $1+\mathcal{H}$,

$$
1+\mathcal{H}=\left(\frac{\cosh u-1}{u^{2}}\right)(3-\cosh u),
$$

is positive. That is, the admissible Fermi coordinates must remain within a circular cylinder about the $Z$ axis such that $\cosh u<3$ or $\rho<\rho_{\max }$, where

$$
\rho_{\max }=\frac{\sqrt{2}}{\Omega} \ln (1+\sqrt{2}) \approx \frac{1.25}{\Omega} .
$$

Expressing the Fermi metric (78) in terms of cylindrical coordinates $(T, \rho, \varphi, Z)$, we find

$$
\begin{aligned}
d s^{2}= & -(1+L) d T^{2}-2 \Omega \rho^{2} F d T d \varphi+d \rho^{2} \\
& +\rho^{2}(1+\mathcal{H}) d \varphi^{2}+d Z^{2} .
\end{aligned}
$$

In this form, the Gödel spacetime region under consideration is stationary and cylindrically symmetric, and the corresponding Killing vectors are $\partial_{T}, \partial_{\varphi}$, and $\partial_{Z}$. It is important to note that the square of the magnitude of the azimuthal Killing vector is $\rho^{2}(1+\mathcal{H})$, which is positive only for $\rho<\rho_{\max }$. Thus for a given $T$ and $Z$, the circle of radius $\rho$ is spacelike within the admissible region, null at $\rho=\rho_{\max }$ and timelike for $\rho>\rho_{\max }$. The admissible re- 
gion therefore excludes closed timelike lines that are known to exist in Gödel spacetime [17,18].

It is interesting to employ Fermi coordinates to study some of the properties of Gödel spacetime. We recall that the source of the Gödel solution has four-velocity $u^{\mu}$ that coincides with the timelike Killing vector in $x^{\mu}=$ $(t, x, y, z)$ coordinates of Eqs. (44) and (45). Under the transformation $\quad x^{\mu} \mapsto \tilde{x}^{\mu}=(T, \rho, \varphi, Z), \quad u_{\mu} \mapsto \tilde{u}_{\mu}=$ $\left(\partial x^{\alpha} / \partial \tilde{x}^{\mu}\right) u_{\alpha}$, so that using $u_{\alpha}=g_{\alpha 0}$ we have

$$
\tilde{u}_{\mu}=-\frac{\partial t}{\partial \tilde{x}^{\mu}}-\sqrt{2} U(x) \frac{\partial y}{\partial \tilde{x}^{\mu}},
$$

which can be simply computed using Eq. (74). For the metric $\tilde{g}_{\mu \nu}$ given by Eq. (83), $\sqrt{-\bar{g}}=(\sqrt{2} \Omega)^{-1} \sinh u$, and the only nonzero components of $\tilde{g}^{\mu \nu}$ are $\tilde{g}^{\rho \rho}=\tilde{g}^{Z Z}=1$ and

$$
\begin{gathered}
\tilde{g}^{T T}=\frac{\chi-3}{\chi+1}, \quad \tilde{g}^{T \varphi}=\tilde{g}^{\varphi T}=-\Omega \frac{\chi-1}{\chi+1}, \\
\tilde{g}^{\varphi \varphi}=\Omega^{2} \frac{\chi^{2}+1}{\chi^{2}-1},
\end{gathered}
$$

where $\chi=\cosh u$. It follows that $\tilde{u}^{\mu}=(1,0,-\Omega, 0)$ in $\tilde{x}^{\mu}$ coordinates or, expressed geometrically, $\partial_{t}=\partial_{T}-\Omega \partial_{\varphi}$.

The Gödel spacetime is of Petrov type D and has five Killing vector fields. In his original paper [17], Gödel already discussed the simple symmetries associated with four Killing vectors, which in terms of $(t, x, y, z)$ coordinates of Eqs. (44) and (45) are given by $\partial_{t}, \partial_{y}, \partial_{z}$, and $\partial_{x}-$ $\sqrt{2} \Omega y \partial_{y}$. The fifth Killing vector has a more complicated form

$$
K=2 \sqrt{2} U^{-1} \partial_{t}-2 \sqrt{2} \Omega y \partial_{x}+\left(2 \Omega^{2} y^{2}-U^{-2}\right) \partial_{y} .
$$

This can be derived directly from the Killing equation; alternatively, one can use the simple Killing vectors in the new forms of the metric given in Eqs. (77), (78), or (83) to find it. For instance, one can use Eqs. (71)-(73) to determine the partial derivatives of $t, x, y$, and $z$ with respect to $v$. The result is

$$
\begin{aligned}
\partial_{v}= & \frac{1}{\Omega}\left(U^{-1}-1\right) \partial_{t}-y \partial_{x} \\
& +\frac{1}{2 \sqrt{2} \Omega}\left[1+\left(2 \Omega^{2} y^{2}-U^{-2}\right)\right] \partial_{y},
\end{aligned}
$$

so that the "new" Killing vector $\partial_{v}$ is expressible in terms of those in the original coordinate system as

$$
2 \sqrt{2} \Omega \partial_{v}=-2 \sqrt{2} \partial_{t}+\partial_{y}+K .
$$

It is remarkable that pursuing such an approach to the symmetries of Gödel spacetime, Obukhov already discovered the coordinate transformations (71)-(73) in Ref. [21].

\section{TIDAL DYNAMICS IN GÖDEL SPACETIME}

Let us now consider the motion of a free test particle in the exact Fermi coordinate system that we have constructed in Sec. IV. Our purpose here is to compare the dynamics in the exact case with that using only the firstorder tidal terms; that is, the solution of the equations of motion based on the metric form (52).

To simplify matters, let us limit our considerations to "radial" motion orthogonal to the $Z$ direction. Specifically, we assume that at $T=0,(X, Y, Z)=\mathbf{0}$ and $\mathbf{V}=\left(V_{0}, 0,0\right)$, where $V_{0} \in[0,1)$. Starting from the exact Fermi metric (83) in cylindrical coordinates, we find the equations of motion

$$
\begin{gathered}
\left(\frac{d \rho}{d T}\right)^{2}=\frac{\chi+1}{3-\chi}-\frac{1}{\Gamma_{0}^{2}}\left(\frac{\chi+1}{3-\chi}\right)^{2}, \\
\frac{d \varphi}{d T}=\Omega \frac{\chi-1}{3-\chi}
\end{gathered}
$$

where, as in Sec. IV, $\chi=\cosh (\sqrt{2} \Omega \rho)$ and $\Gamma_{0}$ is the Lorentz factor corresponding to $V_{0}$. Let $w:=\chi+1$, so that

$$
w=2 \cosh ^{2}\left(\frac{\Omega \rho}{\sqrt{2}}\right) .
$$

For $0 \leq \rho<\rho_{\max }$, we have that $2 \leq w<4$. Equation (89) can be written in terms of $w$ and reduced to quadrature as

$$
\int_{2}^{w} \frac{-1+4 / x}{\sqrt{(x-2)\left[4-\left(2-V_{0}^{2}\right) x\right]}} d x= \pm \sqrt{2} \Omega T .
$$

Next, formulas 2.261 on p. 81 and 2.266 on p. 84 of Ref. [22] can be employed to express the general solution as

$$
\begin{aligned}
& -\sqrt{2} \arcsin W_{1}+\frac{1}{\sqrt{2-V_{0}^{2}}} \arcsin W_{2} \\
& +\frac{\pi}{2}\left(\sqrt{2}-\frac{1}{\sqrt{2-V_{0}^{2}}}\right)= \pm \sqrt{2} \Omega T,
\end{aligned}
$$

where $W_{1}$ and $W_{2}$ are given by

$$
\begin{gathered}
W_{1}=\frac{\left(4+V_{0}^{2}\right)-\left(4-V_{0}^{2}\right) \chi}{V_{0}^{2}(\chi+1)}, \\
W_{2}=\frac{2-\left(2-V_{0}^{2}\right) \chi}{V_{0}^{2}} .
\end{gathered}
$$

It follows from Eq. (89) that in this "radial" motion with zero orbital angular momentum about the $Z$ axis, $\rho$ increases monotonically from zero and after reaching a maximum at 


$$
\hat{\rho}\left(V_{0}\right)=\frac{1}{\sqrt{2} \Omega} \ln \left(\frac{\sqrt{2}+V_{0}}{\sqrt{2}-V_{0}}\right)
$$

returns to the origin in a time-symmetric fashion. For $V_{0}: 0 \rightarrow 1, \hat{\rho}: 0 \rightarrow \rho_{\max }$; that is, a test particle remains within the Fermi frame, while a null ray reaches the null circular boundary. An interesting feature of $\ddot{\rho}$, derived from Eq. (89), should be noted: it vanishes at $\rho=0$ for $V_{0}=1 / \sqrt{2}$, which is the critical speed for relative gravitational motion.

The same kind of motion in the case of first-order tidal terms, metric (52), turns out to be purely radial with the equation of motion

$$
\ddot{\rho}=-\Omega^{2}\left(1-2 \dot{\rho}^{2}\right) \rho
$$
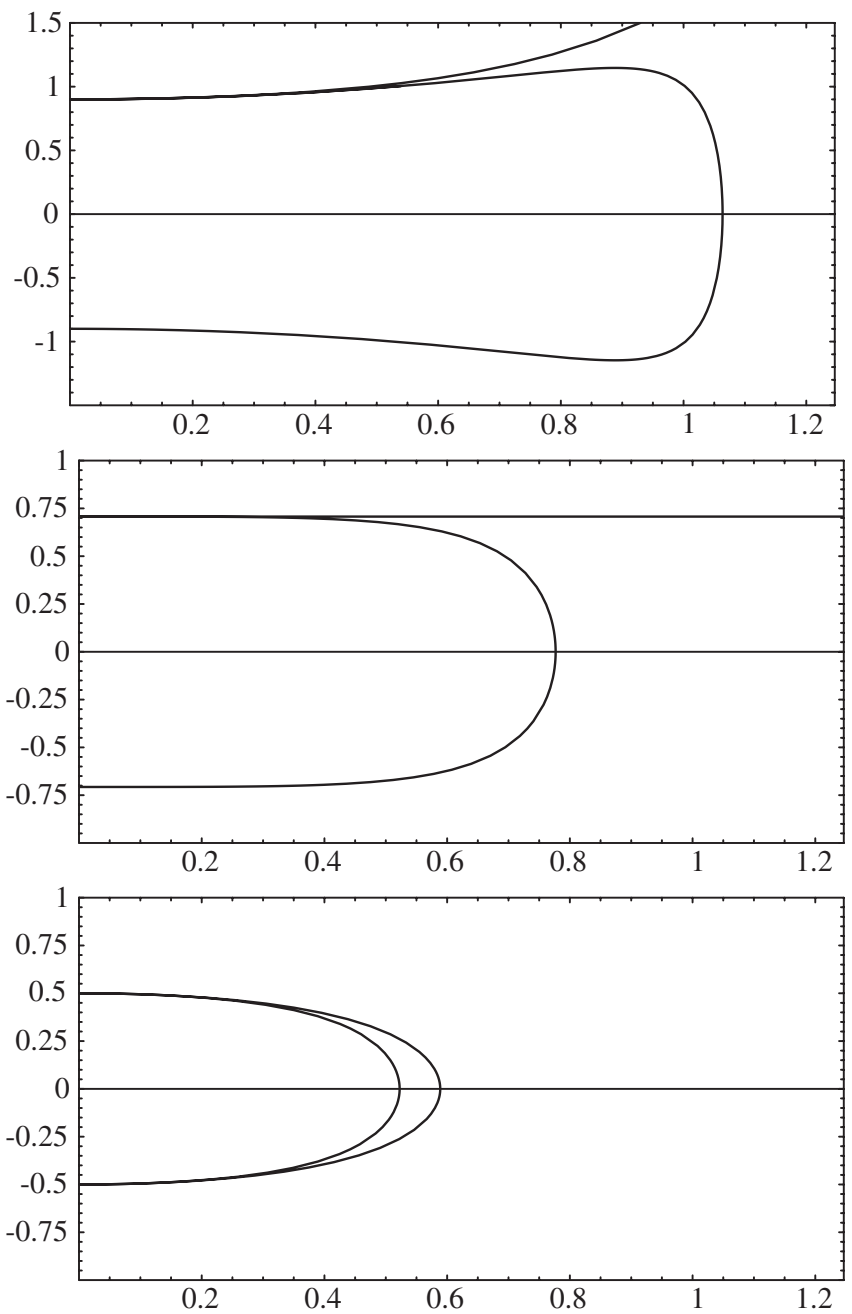

FIG. 3. Plot of $\dot{\rho}$ versus $\Omega \rho, 0 \leq \Omega \rho \leq \sqrt{2} \ln (1+\sqrt{2})$, for $V_{0}=0.5,1 / \sqrt{2}$ and 0.9 . In the top and middle panels, the lower (upper) curves represent the motion according to the exact (approximate) equation of motion in Fermi coordinates. In the top panel, $\dot{\rho}$ monotonically increases in the approximate case. In the bottom panel, the inner curve corresponds to the exact equation of motion. and initial conditions that at $T=0, \rho=0$ and $\dot{\rho}=V_{0}$. This equation is expected to be valid for $\Omega \rho$ sufficiently small compared to unity. Equation (97) can be integrated once and the result is

$$
\dot{\rho}^{2}=V_{c}^{2}-\left(V_{c}^{2}-V_{0}^{2}\right) e^{2 \Omega^{2} \rho^{2}} ;
$$

moreover, the solution of Eq. (98) can be expressed as

$$
\Psi_{+}\left(\sqrt{2} \Omega \rho ; 2 V_{0}^{2}-1\right)= \pm \Omega T .
$$

It is simple to see from the effective potential in Eq. (98) that for $V_{0}>V_{c}$, the particle monotonically accelerates to almost the local speed of light, while for $V_{0}=V_{c}$, the motion is uniform. For $V_{0}<V_{c}$, the motion along any radial axis in the $(X, Y)$ plane is periodic and confined to the interval $\left[-\rho_{0}, \rho_{0}\right]$, where

$$
\rho_{0}=\frac{1}{\sqrt{2} \Omega} \sqrt{\ln \left(\frac{V_{c}^{2}}{V_{c}^{2}-V_{0}^{2}}\right)} .
$$

We note that for $0<V_{0}<V_{c}, \rho_{0}>\hat{\rho}$, while for $V_{0}=0$, $\rho_{0}=\hat{\rho}=0$. These results, together with a comparison with the exact solution, are illustrated in Fig. 3. Analytic estimates for the difference between the exact solution and the approximation can be obtained using Eqs. (93) and (99).

\section{DISCUSSION}

The metrics of de Sitter and Gödel spacetimes in Fermi coordinates can be expressed in infinite series of tidal terms that are uniformly convergent over all of space and time; however, the requirement of (Lichnerowicz) admissibility limits their respective domains of applicability to $0 \leq$ $H R<\pi / 2$ and $0 \leq \Omega \rho<\sqrt{2} \ln (1+\sqrt{2})$. In terms of the motion of free test particles in these domains, it turns out that - for the cases considered in this paper- the firstorder tidal terms already provide good approximations over significant neighborhoods about the origins of these domains as illustrated in Figs. 2 and 3. It is possible to provide detailed analytic estimates of the difference between the geodesic (deviation) equation and the generalized Jacobi equation using the solutions of these equations of relative motion presented in Secs. III and V.

\section{ACKNOWLEDGMENTS}

B.M. is grateful to Valeri Frolov for interesting discussions.

\section{APPENDIX A: CRITICAL SPEED}

Imagine the radial motion of a swarm of particles away from a collapsed configuration as in an astrophysical jet. Relative to a free test observer and in a Fermi coordinate system based on this observer's worldline, free particles starting from the position of the observer and moving ultrarelativistically outward with speed above $V_{c}=1 / \sqrt{2}$ 
decelerate toward the critical speed $1 / \sqrt{2}$. However, free particles moving ultrarelativistically normal to the jet direction accelerate relative to the observer; via collisions with neighboring particles, the corresponding tidal energy can be imparted to the collapsed object's environment. One can in fact envision at the position of the observer a critical velocity cone with its axis along the jet direction and a total opening angle of $2 \theta_{c}$ at its vertex such that $\tan \theta_{c}=\sqrt{2}$. Within this cone free ultrarelativistic particles decelerate relative to the observer, while they accelerate outside the cone. For infrarelativistic motion with speed below $V_{c}=$ $1 / \sqrt{2}$, tidal effects tend to behave more or less as one would generally expect on the basis of Newtonian gravitation theory. That ultrarelativistic gravitational tidal effects could exhibit astrophysically interesting features that would be contrary to Newtonian expectations was first pointed out in Ref. [11] and has been the subject of several recent papers $[12,13]$. It is important to emphasize the general significance of the critical speed for relative motion in accelerated systems and gravitational fields. These results follow from the generalized Jacobi equation that is based on the lowest-order tidal terms; we explain in Appendix B why it is impractical to use exact tidal terms for these astrophysically significant problems.

It is interesting to point out here that in the specific context of (essentially radial) geodesic motion in the exterior Schwarzschild field, the critical speed was already discussed by Hilbert [23]. An examination of the early references to this subject is contained in Ref. [24]. In terms of Schwarzschild coordinates, the critical speed is given by $v_{c}=1 / \sqrt{3}$ as discussed in detail in Ref. [25]. Using a more invariant approach, McVittie [26] derived the critical speed $V_{c}=1 / \sqrt{2}$ for geodesic motion in Schwarzschild spacetime.

Shapiro's observation of the gravitational time delay can be interpreted to imply that light slows down in the gravitational field of a massive body. This is in apparent conflict with the fact that in Newtonian gravity particles speed up as they fall toward a massive object. These ideas are properly integrated in general relativity through the concept of critical speed. In this context, $v_{c}$ and $V_{c}$ have been treated in Ref. [25], which should be consulted for a more detailed treatment of (and further references to) this topic. Finally, we note that the critical speed $v_{c}=1 / \sqrt{3}$ has been discussed recently in connection with the deflection of particles by a radially moving gravitational lens [27].

\section{APPENDIX B: FERMI COORDINATES IN SCHWARZSCHILD SPACETIME}

The purpose of this appendix is to bring out the difficulties encountered in attempts to employ explicit Fermi coordinates in black hole spacetimes. To this end, we imagine in the following treatment the simplest situation of astrophysical interest, namely, purely radial motion in the exterior Schwarzschild gravitational field.
Consider the exterior Schwarzschild spacetime of a spherical source of mass $M$ represented by the metric

$$
\begin{aligned}
d s^{2}= & -\left(1-\frac{2 M}{r}\right) d t^{2}+\left(1-\frac{2 M}{r}\right)^{-1} d r^{2} \\
& +r^{2}\left(d \theta^{2}+\sin ^{2} \theta d \phi^{2}\right)
\end{aligned}
$$

for $r>2 M$. To simplify matters, we limit our treatment to the motion of test particles along a fixed radial direction, which we can choose to be the $z$ axis (i.e. $\theta=0$ ) with no loss of generality.

To construct Fermi coordinates for this two-dimensional world, we must specify a reference observer. Imagine, therefore, a free observer $O:(\bar{t}, \bar{r})$ that starts from $r_{0}>$ $2 M$ at $\bar{t}=0$ and follows an escape trajectory that reaches radial infinity with zero speed. The geodesic equations of motion for this observer are

$$
\frac{d \bar{t}}{d \tau}=\frac{1}{1-\frac{2 M}{\bar{r}}}, \quad \frac{d \bar{r}}{d \tau}=\sqrt{\frac{2 M}{\bar{r}}},
$$

where $\tau$ is the observer's proper time such that $\tau=0$ at $\bar{t}=0$ and $\bar{r}=r_{0}$. The system (B2) can be integrated and the result is

$$
\begin{gathered}
\bar{r}=2 M \cosh ^{2} w, \quad 3 \tau-4 M \cosh ^{3} w=c_{1}, \\
3 \bar{t}-4 M \cosh ^{3} w-12 M\left(\cosh w+\ln \tanh \frac{w}{2}\right)=c_{2},
\end{gathered}
$$

where $c_{1}$ and $c_{2}$ are constants of integration and can be expressed in terms of $r_{0}$ using the initial conditions.

The observer carries an orthonormal parallel-propagated tetrad $\lambda_{(\alpha)}^{\mu}$ along its path. This local frame is given by unit vectors along the temporal and radial directions

$$
\begin{gathered}
\lambda_{(0)}^{\mu}=\left(\left(1-\frac{2 M}{\bar{r}}\right)^{-1}, \sqrt{\frac{2 M}{\bar{r}}}, 0,0\right), \\
\lambda_{(3)}^{\mu}=\left(\sqrt{\frac{2 M}{\bar{r}}}\left(1-\frac{2 M}{\bar{r}}\right)^{-1}, 1,0,0\right),
\end{gathered}
$$

in $(t, r, \theta, \phi)$ coordinates, respectively, as well as $\lambda^{\mu}{ }_{(1)}$ and $\lambda^{\mu}{ }_{(2)}$. The axial symmetry of the spacetime about the $z$ axis implies that a rotational degeneracy exists in the choice of $\lambda^{\mu}{ }_{(1)}$ and $\lambda^{\mu}{ }_{(2)}$. But, once these unit vectors are chosen at $\bar{t}=0$, they are then parallel transported along the path of $O$.

Let us next consider the spacelike geodesics appropriate to the two-dimensional world of radial motion under consideration. They are given by

$$
\frac{d t}{d \sigma}=\frac{\hat{p}}{1-\frac{2 M}{r}}, \quad \frac{d r}{d \sigma}=\epsilon \sqrt{\hat{p}^{2}+1-\frac{2 M}{r}},
$$

where $\hat{p}$ is an integration constant and $\epsilon= \pm 1$. As in 
Fig. 1, we need only those spacelike geodesics that are orthogonal to the reference worldline at $Q$. It follows from $\xi_{\mu} \lambda_{(0)}^{\mu}=0$ that

$$
\hat{p}=\epsilon \sqrt{\frac{2 M}{\bar{r}}} .
$$

Moreover, it follows from Eq. (1) that in this case the Fermi coordinates are

$$
T=\tau, \quad X=Y=0, \quad Z=\epsilon \sigma .
$$

It remains to integrate system (B7) from $Q$ to $P$ for constant $T$. This system can be written as

$$
\frac{d t}{d Z}=\frac{p}{1-\frac{2 M}{r}}, \quad \frac{d r}{d Z}=\sqrt{q^{2}-\frac{2 M}{r}},
$$

where $p=\epsilon \hat{p}$ and $q$ are functions of $T$,

$$
p=\sqrt{\frac{2 M}{\bar{r}}}, \quad q=\sqrt{1+\frac{2 M}{\bar{r}}} .
$$

The solution of system (B10) is simplified if we introduce a new quantity $\zeta$ such that

$$
\sqrt{\frac{r}{2 M}}=\frac{1}{q} \cosh \zeta
$$

Then, we find

$$
\begin{gathered}
\zeta+\frac{1}{2} \sinh (2 \zeta)-\bar{\zeta}-\frac{1}{2} \sinh (2 \bar{\zeta})=\frac{q^{3}}{2 M} Z, \\
\mathcal{F}(\zeta)-\mathcal{F}(\bar{\zeta})+\frac{2 p}{q}(\zeta-\bar{\zeta})=\frac{1}{2 M}[t-\bar{t}(T)-p Z]
\end{gathered}
$$

where $\bar{\zeta}$ is given by

$$
\bar{\zeta}=\cosh ^{-1}\left(\frac{q}{p}\right)
$$

and $\mathcal{F}(\zeta)$ is defined to be

$$
\mathcal{F}(\zeta)=\ln \left(\frac{e^{2 \zeta}-A}{e^{2 \zeta}-B}\right)
$$

Here $A$ and $B$ are functions of $T$ given by

$$
A=1+2 p^{2}+2 p q, \quad B=1+2 p^{2}-2 p q .
$$

Inspection of Eq. (B13) makes it evident that it is not possible to express $\zeta$ explicitly in terms of $T$ and $Z$. It follows that the radial coordinate $r$ in the Schwarzschild metric (B1) cannot be expressed explicitly in terms of $T$ and $Z$. For the explicit expression of the metric in Fermi coordinates, one must resort to expansions in powers of $Z$. It is therefore clear that the transformation $(t, r) \mapsto(T, Z)$ is given only implicitly by Eqs. (B12)-(B17). Taylor expansions can be used to obtain an explicit form for the transformation to Fermi coordinates. We find that

$$
\begin{gathered}
t=\bar{t}(T)+f_{1} Z+\frac{1}{2 !} f_{2} Z^{2}+\frac{1}{3 !} f_{3} Z^{3}+\mathcal{O}\left(Z^{4}\right), \\
r=\bar{r}(T)+Z+\frac{1}{2} \frac{M}{\bar{r}^{2}} Z^{2}-\frac{1}{3} \frac{M}{\bar{r}^{3}} Z^{3}+\mathcal{O}\left(Z^{4}\right),
\end{gathered}
$$

where

$$
\begin{gathered}
f_{1}=\frac{p}{1-p^{2}}, \quad f_{2}=-\frac{1}{\bar{r}} \frac{p^{3}}{\left(1-p^{2}\right)^{2}}, \\
f_{3}=\frac{1}{2 \bar{r}^{2}} \frac{p^{3}\left(4-p^{2}+p^{4}\right)}{\left(1-p^{2}\right)^{3}} .
\end{gathered}
$$

Using transformations (B18) and (B19), we find the relevant metric coefficients in Fermi coordinates

$$
\begin{gathered}
g_{T T}=-1+\frac{2 M}{\bar{r}^{3}} Z^{2}+\mathcal{O}\left(Z^{3}\right), \quad g_{T Z}=g_{Z T}=\mathcal{O}\left(Z^{3}\right), \\
g_{Z Z}=1+\mathcal{O}\left(Z^{3}\right),
\end{gathered}
$$

in agreement with Eqs. (2)-(4), since in this case

$$
R_{T Z T Z}=-\frac{2 M}{\bar{r}^{3}}, \quad \bar{r}=\left(r_{0}^{3 / 2}+\frac{3}{2} \sqrt{2 M} T\right)^{2 / 3} .
$$

We have shown that it is not possible to provide explicit Fermi coordinates for radial motion in the exterior Schwarzschild spacetime. The upshot of our direct approach is just a different approximation scheme for determining the series of tidal terms in Eqs. (2)-(4). In this way, we have also provided the justification for using the generalized Jacobi equation in our previous work [11-13].

\section{APPENDIX C: EXTENT OF VALIDITY OF THE GENERALIZED JACOBI APPROXIMATION}

The generalized Jacobi Eq. (11) is a first-order approximation to the geodesic deviation Eq. (8) in Fermi normal coordinates. The purpose of this appendix is to describe a general method for determining the time scale over which the first-order approximation is valid.

It is convenient to express Eqs. (8) and (11) in dimensionless form; to this end, we introduce $\mathcal{T}:=T / \mathcal{R}$ and $\mathcal{X}:=\mathbf{X} / \mathcal{R}$, where $\mathcal{R}$ is a constant effective radius of curvature of the spacetime under consideration such that $|X|<1$ in the domain of admissibility of Fermi coordinates. In terms of $\mathcal{U}:=(\mathcal{X}, \mathbf{V})$, Eqs. (8) and (11) can be expressed, respectively, as

$$
\begin{gathered}
\frac{d \mathcal{U}}{d \mathcal{T}}=(\mathbf{V}, \mathbf{P}(\mathcal{U})+\mathbf{Q}(\mathcal{U})), \\
\frac{d \tilde{U}}{d \mathcal{T}}=(\tilde{\mathbf{V}}, \mathbf{P}(\tilde{\mathcal{U}}))
\end{gathered}
$$

Here 


$$
\begin{aligned}
P^{i}(\mathcal{X}, \mathbf{V}):= & -\mathcal{R}^{2}\left[{ }^{F} R_{0 i 0 j}+2^{F} R_{i k j 0} V^{k}+\frac{2}{3}\left(3^{F} R_{0 k j 0} V^{i} V^{k}\right.\right. \\
& \left.\left.+{ }^{F} R_{i k j l} V^{k} V^{l}+{ }^{F} R_{0 k j l} V^{i} V^{k} V^{l}\right)\right] \chi^{j}, \\
Q^{i}(\mathcal{X}, \mathbf{V}) & :=-\left[\mathcal{R}\left(\Gamma^{i}{ }_{\alpha \beta}-\Gamma_{\alpha \beta}^{0} V^{i}\right) V^{\alpha} V^{\beta}+P^{i}(\chi, \mathbf{V})\right],
\end{aligned}
$$

where $V^{\alpha}:=d X^{\alpha} / d T=(1, \mathbf{V})$.

To estimate the difference between the solutions of Eqs. (8) and (11), we introduce $\mathcal{Z}:=\mathcal{U}-\tilde{U}$ and note that

$$
\frac{d \mathcal{Z}}{d \mathcal{T}}=J(\mathcal{U})-J(\tilde{\mathcal{U}})+S(\mathcal{U})
$$

where

$$
J(\mathcal{U}):=(\mathbf{V}, \mathbf{P}(\mathcal{U})), \quad S(\mathcal{U}):=(0, \mathbf{Q}(\mathcal{U})) .
$$

The initial conditions for Eqs. (8) and (11) are the same, hence it follows from the integration of Eq. (C5) that

$$
|\mathcal{Z}(\mathcal{T})| \leq \int_{0}^{\mathcal{T}}|J(\mathcal{U})-J(\tilde{\mathcal{U}})| d \mathcal{T}^{\prime}+\int_{0}^{\mathcal{T}}|S(\mathcal{U})| d \mathcal{T}^{\prime}
$$

It is clear from definitions (C3) and (C4) that $\mathbf{Q}$ consists of tidal terms of second, third, and higher orders. Since $|X|<$ 1 and $|\mathbf{V}| \lesssim 1$, we may suppose that $\|D J\| \leq A_{0}$ and $\|$ $S \| \leq B_{0}$ over an interval of interest $[0, \mathcal{T}]$ for some positive constants $A_{0}$ and $B_{0}$. Hence it follows from Eq. (C7) that

$$
|Z(\mathcal{T})| \leq A_{0} \int_{0}^{\mathcal{T}}\left|Z\left(\mathcal{T}^{\prime}\right)\right| d \mathcal{T}^{\prime}+B_{0} \mathcal{T}
$$

By an application of Gronwall's inequality [28], we have the fundamental estimate

$$
|Z(\mathcal{T})| \leq B_{0} \mathcal{T} e^{A_{0} \mathcal{T}},
$$

which implies that

$$
|\chi(\mathcal{T})-\tilde{X}(\mathcal{T})| \leq B_{0} \mathcal{T} e^{A_{0} \mathcal{T}} .
$$

As a measure of the validity of the approximation of $\chi(\mathcal{T})$ by $\tilde{X}(\mathcal{T})$, we can determine the timescale $\mathcal{T}_{\epsilon}$ over which the magnitude of their difference is less than $\epsilon, 0<$ $\epsilon \ll 1$; that is,

$$
B_{0} \mathcal{T}_{\epsilon} e^{A_{0} \mathcal{T}_{\epsilon}}=\epsilon .
$$

Using Lambert's $W$ function, which is the inverse of the function $x \mapsto x e^{x}$, we find

$$
\mathcal{T}_{\epsilon}=\frac{1}{A_{0}} W\left(\frac{A_{0} \epsilon}{B_{0}}\right)
$$

It should be clear that this method provides a general but crude estimate; indeed, sharper estimates may be obtained in specific cases using explicit solutions as in Secs. III and $\mathrm{V}$ of this paper.
[1] E. Fermi, Nuovo Cimento 22, 176 (1921).

[2] J. L. Synge, Relativity: The General Theory (NorthHolland, Amsterdam, 1960).

[3] D. Bini and R. T. Jantzen, Nuovo Cimento B 117, 983 (2002).

[4] A. Lichnerowicz, Théories Relativistes de la Gravitation et de l'Électromagnétisme (Masson, Paris, 1955).

[5] T. Levi-Civita, Math. Ann. 97, 291 (1926).

[6] F. K. Manasse and C. W. Misner, J. Math. Phys. (N.Y.) 4, 735 (1963).

[7] B. Mashhoon, Astrophys. J. 216, 591 (1977).

[8] W.-Q. Li and W.-T. Ni, J. Math. Phys. (N.Y.) 20, 1925 (1979).

[9] M. Ishii, M. Shibata, and Y. Mino, Phys. Rev. D 71, 044017 (2005).

[10] K.-P. Marzlin, Phys. Rev. D 50, 888 (1994).

[11] C. Chicone and B. Mashhoon, Classical Quantum Gravity 19, 4231 (2002).

[12] C. Chicone, B. Mashhoon, and B. Punsly, Int. J. Mod. Phys. D 13, 945 (2004); Phys. Lett. A 343, 1 (2005).

[13] C. Chicone and B. Mashhoon, Classical Quantum Gravity 21, L139 (2004); 22, 195 (2005); Ann. Phys. (Leipzig) 14, 290 (2005); Astron. Astrophys. 437, L39 (2005); Ann.
Phys. (Berlin) 14, 751 (2005); Classical Quantum Gravity 23, 4021 (2006).

[14] Y. Kojima and K. Takami, Classical Quantum Gravity 23, 609 (2006).

[15] D. Bini, C. Cherubini, S. Filippi, and A. Geralico, Classical Quantum Gravity 22, 5157 (2005); D. Bini, A. Geralico, and R. T. Jantzen, Classical Quantum Gravity 22, 4729 (2005); B. Linet, Phys. Rev. D 70, 048101 (2004).

[16] H.-J. Schmidt, Fortschr. Phys. 41, 179 (1993).

[17] K. Gödel, Rev. Mod. Phys. 21, 447 (1949).

[18] W. Kundt, Z. Phys. 145, 611 (1956); S. Chandrasekhar and J. P. Wright, Proc. Natl. Acad. Sci. USA 47, 341 (1961); S.W. Hawking and G.F.R. Ellis, The Large Scale Structure of Space-Time (Cambridge University Press, Cambridge, England, 1973), Chap. 5; H. Stephani, D. Kramer, M. A. H. MacCallum, C. Hoenselaers, and E. Herlt, Exact Solutions of Einstein's Field Equations (Cambridge University Press, Cambridge, England, 2003), 2nd ed.

[19] B. Mashhoon, Phys. Rev. D 11, 2679 (1975).

[20] M. Novello, N. F. Svaiter, and M.E. X. Guimarães, Gen. Relativ. Gravit. 25, 137 (1993). 
[21] Yu. N. Obukhov, in Colloquium on Cosmic Rotation, Berlin, 1998, edited by M. Scherfner, T. Chrobok, and M. Shefaat (Wissenschaft und Technik Verlag, Berlin, 2000), pp. 23-96; astro-ph/0008106.

[22] I.S. Gradshteyn and I. M. Ryzhik, Table of Integrals, Series and Products (Academic Press, New York, 1980).

[23] D. Hilbert, Math. Ann. 92, 1 (1924).

[24] C. H. McGruder, III, Phys. Rev. D 25, 3191 (1982).
[25] B. Mashhoon, Int. J. Mod. Phys. D 14, 2025 (2005).

[26] G.C. McVittie, General Relativity and Cosmology (Chapman and Hall, London, 1956), p. 85.

[27] O. Wucknitz and U. Sperhake, Phys. Rev. D 69, 063001 (2004).

[28] C. Chicone, Ordinary Differential Equations with Applications (Springer-Verlag, New York, 2006), 2nd ed. 